CU-TP-613

August 1993

\title{
Self-intersection, axial anomaly and the string picture of QCD
}

\author{
Ravit Efraty \\ Physics Department, Columbia University \\ New York, NY 10027
}

\begin{abstract}
The leading, planar diagrams of the $1 / N_{c}$ expansion and the usual string description suggest that quarks propagate on the boundary of a two-dimensional world surface. We restrict the quarks to the boundary of the world surface by giving them infinitely large mass on the interior of the surface and zero mass on its boundary and show that in this picture the QCD $\theta$-vacua can be represented by the self-intersection number (or equivalently by the first Chern number of the normal bundle) of the surface.
\end{abstract}

This research was supported in part by the U.S. Department of Energy 


\section{Introduction}

In recent years, an effort has been made to establish the relationship between QCD and strings in order to better understand QCD. Indications that strings might provide an effective description of QCD are seen in the lattice formulation and the $1 / N_{c}$ expansion $[1,2]$. In the limit, $N_{c} \rightarrow \infty$, only planar diagrams, which have exactly the topology of the quantized string with quarks at its ends, contribute. The two-dimensional structure obtained by attaching surfaces to each loop in the planar diagram resembles the world sheet $\Sigma$ of an open string. If QCD behaves like a string theory, we should find string theoretic representations of QCD characteristics. One of the qualitatively important characteristics of QCD is the existence of $\theta$-vacua. The string-candidate associated with the $\theta$-term of QCD, $\frac{\theta}{16 \pi^{2}} \operatorname{tr} F \tilde{F}$, was suggested in $[3,4]$ to be the self-intersection number of the surface, $I(\Sigma)[5]$. In [4] Mazur and Nair introduced fermions in the theory and projected the four-dimensional action onto $\Sigma$. This projection determines the coupling of the fermions to the extrinsic and intrinsic parameters of $\Sigma$. They found that the effect of $\theta$-vacua, represented in the $\mathrm{QCD}$ functional integral by $\exp (i \theta Q)$ where $Q$ is the instanton number, can be represented in the string description by $\exp \left(i \theta C_{1}(\Sigma)\right)$ where $C_{1}(\Sigma)$ is the first Chern number of the normal bundle to $\Sigma$. However in the large $N_{c}$-limit description or the usual string description the quarks are constrained to the boundary of the twodimensional surface, and in [4] the quarks live on the entire surface. Therefore a more accurate description of QCD would require a modified model. In this work we restrict the quarks to the boundary by giving them infinitely large mass on the interior of the surface and zero mass on the boundary. We calculate the axial anomaly for these quarks with a coordinate-dependent mass and obtain a similar relation between the first Chern number of the surface and the total change of the axial charge, $\Delta Q^{5}$.

\section{Short review}

Following Mazur and Nair [4], we begin with the four-dimensional free fermion Lagrangian 


$$
\mathcal{L}=\bar{\Psi} \gamma^{a}\left(\partial_{a}-\frac{1}{8} w_{a}^{\alpha \beta}\left[\gamma_{\alpha}, \gamma_{\beta}\right]\right) \Psi
$$

where we have pulled back quantities from $\Sigma$ to $M$ and $w_{a}^{\alpha \beta}$ is the spin connection. $M$ in general is a Riemann surface with real coordinates $\xi_{a}(a=1,2)$. The image of $M$ in $\mathcal{R}^{4}$ is the surface $\Sigma$. More than one point in $M$ can be mapped to the same point in $\mathcal{R}^{4}$ corresponding to self-intersections of $\Sigma$. Thus the mapping $X^{\mu}(\xi): M \rightarrow \mathcal{R}^{4}$ is, in general, only an immersion. $w_{a}^{\alpha \beta}$ can be expressed in terms of the tangent and normal vectors of the immersion $X^{\mu}(\xi)$ to give the Lagrangian for quarks on $M$. The tangent vectors are $t_{a}^{\mu}=\partial X^{\mu} / \partial \xi^{a}$ and the normal vectors are orthogonal to the tangents at the image points on $\Sigma$. The immersion of $M$ in $\mathcal{R}^{4}$ defines the tangent and normal bundles on $M . A_{a A B}$ is the $S O(2)$ connection for the normal bundle. The extrinsic curvature $K_{a b}^{A}$ is given by the equation

$$
\partial_{a} \partial_{b} X^{\mu}=\Gamma_{a b}^{c} \partial_{c} X^{\mu}+K_{a b}^{A} n_{A}
$$

where $\Gamma_{a b}^{c}$ is the usual Christoffel symbol for the induced metric, $g_{a b}=\partial X^{\mu} / \partial \xi^{a} \partial X^{\mu} / \partial \xi^{b}$. The frame fields on $M, e_{a}^{\hat{a}}$, are given by $e_{a}^{\hat{a}} e_{\hat{a} b}=g_{a b}$. The target space is $\mathcal{R}^{4}$, thus the zerocurvature spin connection is

$$
w_{a}^{\alpha \beta}=t_{a}^{\nu} E^{\alpha \mu} \nabla_{\nu} E^{\beta \mu}
$$

where $E^{\alpha \mu}$ decomposes as follows

$$
E^{\alpha \mu}=\left(n^{A \mu}, t_{\hat{a}}^{\mu} e^{a \hat{a}}\right)
$$

Hence the Lagrangian for fermions on $M$ may be written as

$$
\mathcal{L}=\bar{\Psi} \gamma^{a}\left(\partial_{a}-\frac{1}{8} \Gamma_{a b}^{c}\left[\gamma^{b}, \gamma_{c}\right]+\frac{1}{8} A_{a A B}\left[\gamma^{A}, \gamma^{B}\right]-\frac{1}{4} K_{a b}^{A}\left[\gamma_{b}, \gamma_{A}\right]\right) \Psi
$$

Note that this Lagrangian only contains geometric information. The strings description of QCD should incorporate confinement, thus to obtain a purely geometrical coupling for color singlet combinations there should be an extra factor of $1 / N_{c}$ in the couplings of $K_{a b}^{A}$ 
and $A_{a A B}$ to quarks. This factor arises naturally in the large $N_{c}$-limit of QCD since the quark contribution is smaller by a factor of $1 / N_{c}$ relative to the gluon contribution. Thus the Lagrangian for quarks on $M$ can be taken to be

$$
\mathcal{L}=\bar{q}^{i} \gamma^{a} D_{a} q_{i}
$$

where $D_{a}$ is given by

$$
D_{a}=\partial_{a}-\frac{1}{8} \Gamma_{a b}^{c}\left[\gamma^{b}, \gamma_{c}\right]+\frac{1}{8 N_{c}} A_{a A B}\left[\gamma^{A}, \gamma^{B}\right]-\frac{1}{4 N_{c}} K_{a b}^{A}\left[\gamma_{b}, \gamma_{A}\right]
$$

The standard heat kernel method can be used to obtain the axial anomaly associated with the covariant derivative $D_{a}$. The functional trace of $\gamma_{5}$, the quantity equal to the instanton number in four-dimensional QCD, was found to be [4]

$$
\operatorname{Tr} \gamma_{5}=\frac{1}{N_{c}} C_{1}(\Sigma)
$$

where $C_{1}(\Sigma)$, the first Chern number, is related to the self intersection number $I(\Sigma)$ by

$$
\frac{1}{2} C_{1}(\Sigma)=I(\Sigma) \equiv-\frac{1}{16 \pi} \int d^{2} \xi \sqrt{g} g^{a} b \nabla_{a} t^{\mu \nu} \nabla_{b} \tilde{t}^{\mu \nu},
$$

and has the simple form

$$
C_{1}(\Sigma)=\frac{1}{2 \pi} \int_{M} \operatorname{tr} F
$$

$F$ is the field strength tensor for the $S O(2)$ normal bundle connection and $t^{\mu \nu}=\frac{\epsilon^{a b}}{\sqrt{g}} t_{a}^{\mu} t_{b}^{\nu}$.

A complete analogy with QCD is lacking here because in this picture the quarks live on the two-dimensional surface, rather then propagating only on the boundary as is required by the leading order diagrams of the $1 / N_{c}$ expansion and by the usual string description. To provide a better analogy we instead choose a regularization scheme which allows us to compute the anomaly in two dimensions, while restricting the quarks to the boundary of the surface. 


\section{Regularization scheme}

We give the quarks zero mass on the boundary and infinitely large mass inside the surface. We shall Calculate the propagator for these coordinate-dependent mass quarks and prove that they are indeed restricted to the boundary. For simplicity we take space to be flat with the spatial direction $\sigma$ varying between $-l$ and $l . m(\sigma)$, the quark mass, is chosen to have the simple form:

$$
m(\sigma)= \begin{cases}0, & -l \leq \sigma \leq-a \text { region } \mathrm{I} \\ m_{0}, & -a<\sigma<a \text { region } \mathrm{II} \\ 0, & a \leq \sigma \leq l \text { region III }\end{cases}
$$

We are interested in the limit $m_{0} \rightarrow \infty$. We shall assume that the gauge field of interest, viz. $\left(F_{a b}\right)_{A B}$, the field-strength for $A_{a A B}$, is slowly varying on the scale of $(l-a)$.

\section{Calculating the anomaly for a coordinate-dependent mass}

In order to derive an equation similar to equation (3) we calculate the quantity $\Delta Q^{5}$ given by

$$
\Delta Q^{5}=\int \partial_{\mu} J^{5 \mu} d^{2} \xi
$$

where $J^{5 \mu}$ is the neutral gauge invariant axial current, $J^{5 \mu}=\bar{\Psi} \gamma^{\mu} \gamma^{5} \Psi$, and

$$
\partial_{\mu} J^{5 \mu}=\mathcal{A}+2 i m(x) \bar{\Psi}(x) \gamma^{5} \Psi(x)
$$

where $\mathcal{A}$ is the anomalous term. There are different ways to calculate the axial anomaly. The perturbative method is best suited to our choice of mass. The two-dimensional axial anomaly stems from the two-point function

$$
T_{\mu \nu}^{5}=\left\langle T\left(J_{\mu}(y) J_{\nu}^{5}(x)\right)\right\rangle
$$

For a particle with constant mass, $m$, requiring $J_{\mu}^{5}(x)$ to be gauge invariant results in an anomalous term, $\rho_{2}$ : 


$$
\rho_{2}=-\frac{e}{2 \pi} \epsilon^{\mu \nu} F_{\mu \nu}(x)
$$

Since we want to calculate $q^{\nu} T_{\mu \nu}^{5}$ in a spatially confined space we choose a cutoff regularization. We are also interested in the limit $m_{0} \rightarrow \infty$, thus we choose the cutoff $\Lambda$ and the fixed mass $m_{0}$ such that

$$
\Lambda<m_{0}
$$

We next compute the propagator for $m=m(\sigma)$. In view of equation (9) we search for the solution to the Dirac equation:

$$
(\gamma \cdot \partial-m(\sigma)) \Psi(\sigma, t)=0
$$

with $|E|<\Lambda<m_{0}$ which satisfy the boundary conditions required for a self-adjoint hamiltonian, viz:

$$
\begin{array}{r}
\Psi_{\mathrm{I}}^{*}(-l) \gamma^{0} \gamma^{1} \Psi_{\mathrm{I}}(-l)=0 \\
\Psi_{\mathrm{III}}^{*}(l) \gamma^{0} \gamma^{1} \Psi_{\mathrm{III}}(l)=0
\end{array}
$$

and the matching conditions at $\sigma= \pm a$, where $\Psi_{i}$ is the solution in region $i$. Separation of the time-variable requires a two-phase ansatz:

$$
\Psi=e^{-i E t} \Psi^{(+)}(\sigma)+e^{i E t} \Psi^{(-)}(\sigma)
$$

The static functions $\Psi^{ \pm}(\sigma)$ satisfy

$$
\pm E \Psi^{( \pm)}(\sigma)=(\alpha p+\beta m) \Psi^{( \pm)}(\sigma)
$$

Our representation for the Dirac matrices is $\alpha=\sigma^{2}, \beta=\sigma^{1}$. Implementing the matching conditions and requiring a normalizable solution when $m_{0} \rightarrow \infty$, while fixing the wave function in region III (or I) gives a solution of exponentially decaying wave functions in regions I (or III) and II, but an oscillating wave function in region III (or I). The zero flux condition at $x=l$ gives quantization of energy 


$$
2 k_{n}(l-a)+\varphi=2 \pi n \quad\left\{n \in \mathcal{Z}:\left|k_{n}\right|<\Lambda\right\}
$$

where $k^{2}=E^{2}$ and $\sin \varphi=\frac{k}{m_{0}}$.

For large $m_{0}, \sin \varphi \approx \varphi$ gives

$$
k_{n}=\frac{\pi n}{(l-a)+\frac{1}{2 m_{0}}} \quad-N \leq n \leq N
$$

where

$$
|N| \leq \frac{\Lambda}{\pi}\left(\frac{1}{2 m_{0}}+(l-a)\right)
$$

Fixing $\Psi_{\mathrm{I}}$ instead of $\Psi_{\text {III }}$ yields

$$
2 k_{n}(l-a)+\varphi=2 \pi(n+1 / 2) \quad\left\{n \in \mathcal{Z}:\left|k_{n}\right|<\Lambda\right\} .
$$

The other boundary condition is automatically fulfilled in the large $m_{0}$ limit. The propagator is constructed from $\Psi^{( \pm)}(x)$

$$
\begin{aligned}
S\left(x, x^{\prime}\right)= & -i \theta\left(t^{\prime}-t\right) \sum_{n=-N}^{N} e^{-i E_{n}\left(t^{\prime}-t\right)} \Psi_{n}^{(+)}\left(x^{\prime}\right) \bar{\Psi}_{n}^{(+)}(x) \\
& +i \theta\left(t-t^{\prime}\right) \sum_{n=-N}^{N} e^{i E_{n}\left(t^{\prime}-t\right)} \Psi_{n}^{(-)}\left(x^{\prime}\right) \bar{\Psi}_{n}^{(-)}(x) .
\end{aligned}
$$

For $k_{n}$ satisfying (12) the propagator in regions I and II decays exponentially. Note that in region III the propagator describes a Dirac particle in an infinite square potential well of width $(l-a)+\frac{1}{2 m_{0}}$.

The propagator of a massless fermion in an infinite square potential well of width $(l-a)$, denoted by $S^{0}\left(x^{\prime}, x\right)$, is given by equation (14) with

$$
\Psi_{n}^{(+)}\left(x^{\prime}\right) \bar{\Psi}_{n}^{(+)}(x)=-\frac{1}{l-a}\left(\begin{array}{cc}
\frac{k_{n}}{E_{n}} \sin k_{n}\left(x^{\prime}-l\right) \cos k_{n}(x-l) & \sin k_{n}\left(x^{\prime}-l\right) \sin k_{n}(x-l) \\
\cos k_{n}\left(x^{\prime}-l\right) \cos k_{n}(x-l) & \frac{k_{n}}{E_{n}} \cos k_{n}\left(x^{\prime}-l\right) \sin k_{n}(x-l)
\end{array}\right)
$$

where 


$$
k_{n}=\frac{\pi n}{l-a} \quad N^{0} \leq \frac{\Lambda}{\pi}(l-a) \quad-N^{0} \leq n \leq N^{0} .
$$

$\Psi_{n}^{(-)}\left(x^{\prime}\right) \bar{\Psi}_{n}^{(-)}(x)$ is obtained from (15) by replacing $E_{n}$ with $-E_{n} . S^{0}\left(x^{\prime}, x\right)$ does not have the usual $x^{\prime}-x$ dependence. This is not surprising since in confining the quarks we have lost translational invariance. It is straightforward to check that $S^{0}\left(x^{\prime}, x\right)$ is indeed the propagator. The propagator corresponding to a well of width $(l-a)+\frac{1}{2 m_{0}}$ differs from $S^{0}\left(x^{\prime}, x\right)$ by terms of order $\frac{1}{m_{0}}$ which appear in the normalization of the wave function, $\Psi_{\text {III }}$

$$
|A|^{2}=\frac{1}{4(l-a)}\left(1-\frac{1}{(l-a) 2 m_{0}}\right)
$$

and in the argument of the trigonometric functions

$$
k_{n}=\frac{\pi n}{2(l-a)}\left(1-\frac{1}{(l-a) 2 m_{0}}\right) .
$$

(Though the interval in equation (12) appears different than that in equation (16), we are free to choose $N=N^{0}$.) When computing $T_{\mu \nu}^{5}$ in region III, no additional factors of $m_{0}$ appear. This insures that the $m_{0} \rightarrow \infty$ limit gives the same expression for $T_{\mu \nu}^{5}$ as in the massless case. Thus we find for region III:

$$
\mathcal{A}=\rho_{2}
$$

A similar argument for $k_{n}=\frac{\pi(n+1 / 2)}{(l-a)+1 / 2 m_{0}}$ gives the usual two-dimensional anomaly in region I. In region II, using the cutoff regularization, the propagator between $\sigma$ and $\sigma^{\prime}$ decays exponentially. Therefore all diagrams in that region vanish in the large $m_{0}$ limit. Also all matrix elements of $J^{5}=m_{0} \bar{\Psi} \gamma^{5} \Psi$ vanish in this regularization scheme since the factor of $m_{0}$ cannot compensate for the exponential decay. This gives in region II,

$$
\partial_{\mu} J^{5 \mu}=0
$$

which is expected. A simple way to see this is to recall that for a massive fermion in two dimensions we have 


$$
\partial_{\mu} J^{5 \mu}(x)=\rho_{2}+2 i m \bar{\Psi}(x) \gamma^{5} \Psi(x)
$$

When $m \rightarrow \infty$ the matrix elements of $\bar{\Psi} \gamma^{5} \Psi$ behave like $\frac{1}{m}$, resulting in a non-vanishing remainder for $2 i m \bar{\Psi} \gamma^{5} \Psi$. In fact this remainder is exactly $-\rho_{2}$. Thus we get for a heavy fermion

$$
\lim _{m \rightarrow \infty} \partial_{\mu} J^{5 \mu}(x)=0
$$

which is the same result as (18).

(To understand this better, recall [6] that another way to assure gauge invariance is by introducing a Pauli-Villars regulator field $\psi$ with mass $M$ and a regulated axial current. Then $\partial_{\mu} J^{5 \mu}(x)=2 i m \bar{\Psi}(x) \gamma^{5} \Psi(x)-2 i M \bar{\psi}(x) \gamma^{5} \psi(x)$. When $M \rightarrow \infty$ the regulator field contribution gives the axial anomaly.)

To summarize

$$
\partial_{\mu} J^{5 \mu}= \begin{cases}\rho_{2}, & -l \leq \sigma \leq-a \\ 0, & -a<\sigma<a \\ \rho_{2}, & a \leq \sigma \leq l\end{cases}
$$

\section{Conclusion}

The total change of the axial charge, $\Delta Q^{5}$, constructed from the axial current is

$$
\Delta Q^{5}=\frac{1}{N_{c}} \int d t \int_{\mathrm{I}+\mathrm{III}} d \sigma \rho_{2}
$$

where the same argument that led to the covariant derivative of equation (3) results in a coefficient of $1 / N_{c}$. It is this quantity that we want to relate to the first Chern number or to the self-intersection number of the surface $\Sigma$. (In [4] a relation to the functional trace of $\gamma^{5}$ emerges naturally from the functional integral formalism.) In equation $(4) C_{1}(\Sigma)$ is expressed as an integral of the field strength tensor, $F_{a b A B}$. Since the self-intersection number is a topologically invariant quantity of the surface $\Sigma$, given a connection configuration $A_{a A B}$, we can smoothly deform it to $A_{a A B}^{\prime}$, such that 


$$
\frac{1}{8 \pi} \int \epsilon^{A B} \epsilon^{a b}\left(F_{a b}\right)_{A B} d^{2} \xi=\frac{1}{8 \pi} \int \epsilon^{A B} \epsilon^{a b}\left(F_{a b}^{\prime}\right)_{A B} d^{2} \xi
$$

We can choose this deformation to be the one for which the field strength tensor $F_{a b A B}$ vanishes in region II, i.e. where $m(\sigma) \neq 0$. This insures that the normal bundle is trivial in region II hence only regions I and III contribute to the self-intersection number. Thus we can write

$$
C_{1}(\Sigma)=2 I(\Sigma)=\frac{1}{8 \pi} \int_{\mathrm{I}+\mathrm{III}} \epsilon^{A B} \epsilon^{a b}\left(F_{a b}\right)_{A B} d^{2} \xi
$$

The integrand is exactly $\rho_{2}$. In both equations (20) and (21), the area of integration, which is the 'dynamic' area, is limited to a strip along the boundary where the quarks live. This allows us to relate the QCD topological charge to the first Chern number in the string description

$$
\frac{1}{N_{c}} C_{1}(\Sigma)=\Delta Q^{5}
$$

in the same manner as in ref. [4], but for a more accurate picture in which the quarks are restricted to the boundary.

Note that if we deform $A_{a A B}$ such that a self-intersection occurs in region II, the gauge fields are no longer varying slowly on the scale of $(l-a)$. This must be taken into account when deriving the anomaly (e.g. requiring a more careful treatment of the boundaries between the regions). Presumably, a non-vanishing divergence of the axial current in region II will result and equation (22) will still be valid.

\section{Acknowledgements}

It is a pleasure to thank V. P. Nair for discussions at every stage of this work and a critical reading of the manuscript.

\section{References}

[1] G. 't Hooft, Nucl. Phys. B72 (1974) 461; B75 (1974) 461. 
[2] V. A. Kazakov, Sov. Phys. JETP 58(6) (1983) 1096.

[3] A. P. Balachandran, F. Lizzi and G. Sparano, Nucl. Phys. B263 (1986) 608. A. Polyakov, Nucl. Phys. B268 (1986) 406.

[4] P. O. Mazur and V. P. Nair, Nucl. Phys. B284 (1986) 146.

[5] For general background see M. Spivak, A Comprehensive introduction to differential geometry, (Publish of Perish, Inc, Berkeley, 1979).

[6] R. Jackiw in Current algebra and anomalies by S. B. Treiman, R. Jackiw, B. Zumino and E. Witten, (Princeton University Press, Princeton, New Jersey, 1985). 